\title{
UNIVERSAL ABELIAN COVERS OF SURFACE SINGULARITIES
}

\author{
WALTER D. NEUMANN AND JONATHAN WAHL
}

\begin{abstract}
We discuss the evidence for and implications of a conjecture that the universal abelian cover of a $\mathbb{Q}$-Gorenstein surface singularity with finite local homology (i.e., the singularity link is a $\mathbb{Q}$-homology sphere) is a complete intersection singularity.
\end{abstract}

\section{HISTORY}

Our story starts with a result of Felix Klein. The icosahedral group $I \subset \mathrm{SO}(3)$ is the group of orientation preserving symmetries of a regular icosahedron in $\mathbb{R}^{3}$. The binary icosahedral group $I^{\prime} \subset \mathrm{SU}(2)$ is the inverse image of $I$ under the double cover $\mathrm{SU}(2) \rightarrow \mathrm{SO}(3)$. The quotient $\mathbb{C}^{2} / I^{\prime}$ is a complex variety with an isolated singularity at 0 . Felix Klein showed that

$$
\mathbb{C}^{2} / I^{\prime} \cong V(2,3,5),
$$

where $V(p, q, r)$ is the so-called Brieskorn-Pham variety

$$
V(p, q, r):=\left\{\left(z_{1}, z_{2}, z_{3}\right) \in \mathbb{C}^{3}: z_{1}^{p}+z_{2}^{q}+z_{3}^{r}=0\right\} .
$$

As the reader probably knows, the binary icosahedral group is intimately related to the triple $(2,3,5)$. The non-cyclic finite subgroups of $\mathrm{SU}(2)$ are the binary dihedral and polyhedral groups, and they are classified by the Platonic triples $(p, q, r)$ : triples of integers satisfying

$$
1<p \leq q \leq r, \quad \frac{1}{p}+\frac{1}{q}+\frac{1}{r}>1 .
$$

These are the triples

$$
(2,2, n), n \geq 2 ; \quad(2,3,3),(2,3,4),(2,3,5) .
$$

For a Platonic triple $(p, q, r)$ the group of isometries of the 2-sphere generated by reflections in the sides of a spherical triangle with angles $\frac{\pi}{p}, \frac{\pi}{q}, \frac{\pi}{r}$ is the $(p, q, r)$ triangle group. Its orientation preserving subgroup $\Delta(p, q, r)$ is the dihedral group of order $2 n$ for $(p, q, r)=(2,2, n)$, while $\Delta(2,3,3), \Delta(2,3,4)$ and $\Delta(2,3,5)$ are the tetrahedral, octahedral and icosahedral groups. They are subgroups of $\mathrm{SO}(3)$, and on lifting to $\mathrm{SU}(2)$ we get the corresponding binary groups $\Delta^{\prime}(p, q, r)$; these give all finite non-cyclic subgroups of $\mathrm{SU}(2)$.

Key words and phrases. Gorenstein surface singularity, complete intersection singularity. Research supported under NSF grant no. DMS-0083097.

Research supported under NSA grant no. MDA904-98-1-0016. 
It is thus natural to guess that the relationship $V(2,3,5) \cong \mathbb{C}^{2} / \Delta^{\prime}(2,3,5)$ extends to the other Platonic triples, but this is not correct, as the following table shows:

$$
\begin{aligned}
V(2,3,5) & \cong \mathbb{C}^{2} / \Delta^{\prime}(2,3,5) \\
V(2,3,4) & \cong \mathbb{C}^{2} / \Delta^{\prime}(2,3,3) \\
V(2,3,3) & \cong \mathbb{C}^{2} / \Delta^{\prime}(2,2,2) \\
V(2,2, n) & \cong \mathbb{C}^{2} / C_{n} .
\end{aligned}
$$

(Here, $C_{n}$ is the cyclic group of order $n$.)

The correct relationship is, in fact,

$$
V(p, q, r) \cong \mathbb{C}^{2} /\left[\Delta^{\prime}(p, q, r), \Delta^{\prime}(p, q, r)\right]
$$

where $[G, G]$ denotes the commutator subgroup of a group $G$. In other words, $V(p, q, r)$ is the universal abelian cover of $\mathbb{C}^{2} / \Delta^{\prime}(p, q, r)$ (this is literally true after one removes the singular points from each space, where the map is ramified).

This relationship was shown to extend to the euclidean and hyperbolic triangle groups by J. Milnor [4] and then to groups arising from general polygonal reflection groups in [6]. However, the connection with reflection groups turned out to be a red herring, as was shown in [8]. The essential fact appeared to be that the varieties in question were weighted homogeneous (i.e., admit effective $\mathbb{C}^{*}$-actions):

Theorem 1.1 ([8]). Let $(X, o)$ be a weighted homogeneous normal surface singularity whose link $M$ (boundary of a closed regular neighborhood) is a $\mathbb{Q}$-homology sphere $\left(\right.$ i.e., $\left.H_{1}(M ; \mathbb{Q})=0\right)$. Then the universal abelian cover $(\tilde{X}, o)$ of $(X, o)$ is a Brieskorn-Pham complete intersection.

A Brieskorn-Pham complete intersection is a surface given by $n-2$ equations

$$
a_{1 j} z_{1}^{p_{1}}+\cdots+a_{n j} z_{n}^{p_{n}}=0, \quad j=1, \ldots, n-2,
$$

where $p_{1}, \ldots, p_{n}$ are fixed integers $\geq 2$ and the $a_{i j}$ are sufficiently general coefficients. The exponents $p_{i}$ in this theorem are, in fact, the orders of the isotropy groups of the action of $\mathbb{C}^{*}$ on $X$.

The story does not stop here. One of us (J.W.) suspected that "weighted homogeneous" was also a red herring, and finally persuaded the other that this might be so. We were nevertheless surprised at how general the phenomenon appears to be.

Before describing this in more detail, we would like to stress why a result of this type should be surprising. First, while $\tilde{X}$ in Theorem 1.1 is easily shown to be Gorenstein, it is well-known that being a complete intersection is a much stronger property, and there are no general results we know of which distingush the two. Second, among the weighted homogeneous complete intersections, there are many which are not even of the same topological type as a Brieskorn-Pham one.

There are two obvious necessary conditions for the universal abelian cover of a singularity to be a complete intersection. In this situation, we need to consider $(X, o)$ as a germ of a singularity; we may thus assume $X$ is a regular neighborhood of $o$. The maximal unramified abelian cover of $X-\{o\}$ is a Galois cover with covering transformation group $H_{1}(M ; \mathbb{Z})$, where $M$ is the link of the singularity. If this is a finite cover, then it can always be completed by adding a single point to give a new germ and a map $(\tilde{X}, o) \rightarrow(X, o)$, which is what we mean by the universal abelian cover of $(X, o)$. The two necessary conditions are: 
1. ( $\mathbb{Q}$-homology sphere link). The universal abelian cover must exist. That is, $H_{1}(M ; \mathbb{Z})$ must be finite, or $M$ is a $\mathbb{Q}$-homology sphere.

2. ( $\mathbb{Q}$-Gorenstein). $(X, o)$ should be $\mathbb{Q}$-Gorenstein, that is the dualizing sheaf $\omega_{X}$ has finite order, or, equivalently, for some $n>0, \Gamma\left(X-\{o\}, \omega_{X}^{\otimes n}\right)$ is a free $\mathcal{O}_{X}$-module. Alternatively, $(X, o)$ is the quotient of some Gorenstein singularity by a finite group acting freely off the singular point.

It should also be noted that the Gorenstein condition not only limits the topology, but in the "moduli space" of all singularities with a given topology, non-Gorenstein singularities are nearly always generic (Laufer, in [3], shows the only exceptions are rational double points or minimally elliptic singularities).

Conjecture 1. Let $(X, o)$ be a $\mathbb{Q}$-Gorenstein singularity whose link is a $\mathbb{Q}$-homology sphere. Then the universal abelian cover of $(X, o)$ is a complete intersection singularity.

We have already said why this conjecture is surprising. In the next section we describe what the complete intersection equations conjecturally look like; these "splice diagram equations" generalize the Brieskorn-Pham part of Theorem 1.1. The possible topologies of normal surface singularities have been known for decades (through the work of Grauert and Waldhausen, see e.g., [7]), but it is nevertheless an extremely difficult problem in general to give an explicit algebraic description for a singularity with given topology. It has so far been done only for limited types of topology. In contrast, our conjectures would give explicit algebraic descriptions for a remarkably broad class of topologies.

The next section gives a series of (increasingly speculative) conjectures that refine our main Conjecture 11. Fuller details concerning these conjectures and partial results described here will be published elsewhere.

\section{The Conjectures}

Let $(X, o)$ be a normal surface singularity germ and $M$ its link, that is, the boundary of a regular neighborhood of $o$ in $X$. We shall assume throughout this section that $M$ is a $\mathbb{Q}$-homology sphere.

Let $\pi: \bar{X} \rightarrow X$ be a good resolution. "Good" means that the exceptional divisor $D=\pi^{-1}(o)$ has only normal crossings. The $\mathbb{Q}$-homology sphere condition is equivalent to $D$ being rationally contractible; that is,

- each component of $D$ is a smooth rational curve;

- the dual resolution graph $\Gamma$ (the graph with a vertex for each component of $D$ and an edge for each intersection of two components) is a tree.

We weight each vertex $v$ of $\Gamma$ by the self-intersection number $E_{v} \cdot E_{v}$ of the corresponding component $E_{v}$ of $D$. The intersection matrix for $\Gamma$ is the matrix $A(\Gamma)$ with entries $a_{v w}=E_{v} \cdot E_{w}$, that is,

$$
\begin{aligned}
& a_{v w}=1 \quad \text { if } v \neq w \text { and } v \text { and } w \text { are joined by an edge } \\
& a_{v w}=0 \quad \text { if } v \neq w \text { and } v \text { and } w \text { are not joined by an edge } \\
& a_{v v}=E_{v} \cdot E_{v}
\end{aligned}
$$

It is well known that $A(\Gamma)$ is negative-definite and its cokernel (also called the discriminant group) is $H_{1}(M)$. In particular,

$$
d(\Gamma):=\operatorname{det}(-A(\Gamma))
$$


is the order of $H_{1}(M)$. By a fundamental result of [8], with a few exceptions $A(\Gamma)$ determines and is determined by the topology of $\mathrm{M}$.

To write down equations of the universal abelian cover, we associate another combinatorial object to the weighted dual graph $\Gamma$ - a splice diagram [1]. When $A(\Gamma)$ is unimodular (i.e., $\mathrm{M}$ is an integral homology sphere), this association can be

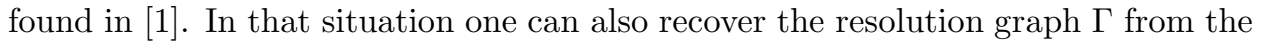
splice diagram, but without unimodularity one usually cannot - many resolution graphs can share the same splice diagram.

Definition 2.1 (Splice diagram of a rationally contractible graph). Let $\Delta$ be the tree obtained by replacing each maximal string in $\Gamma$ by a single edge (a string is a simple path in $\Gamma$ whose interior is open in $\Gamma$ ). Thus $\Delta$ is homeomorphic to $\Gamma$ but has no vertices of valency 2 . At each node $v$ (vertex of valency $\geq 3$ ) of $\Delta$ we introduce weights on the incident edges $e$ as follows. Let $\Gamma_{v e}$ be the subgraph of $\Gamma$ cut off by the edge of $\Gamma$ at $v$ in the direction of $e$ as in the following picture. The corresponding weight is then $d_{v e}:=d\left(\Gamma_{v e}\right)$.

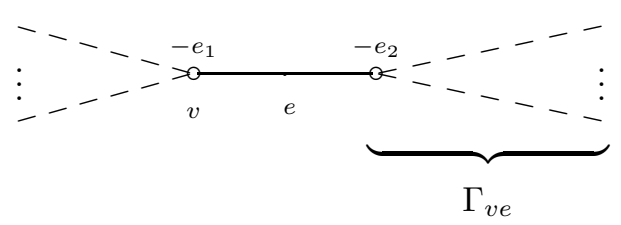

For example, the following is a resolution graph and its splice diagram.

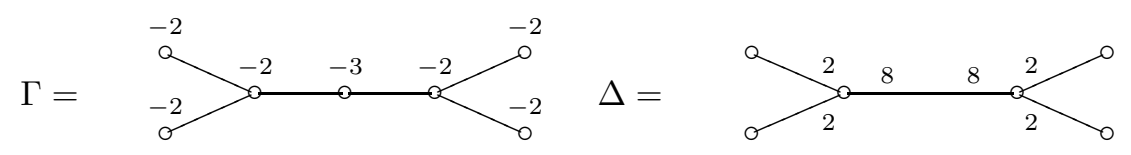

The weight 8 on the left node of $\Delta$ is $d\left(\Gamma_{v e}\right)$ with

$$
\Gamma_{v e}=\stackrel{-3}{-2}
$$

We will associate equations to every node of the splice diagram; some preparation is needed. An end of $\Delta$ is a vertex of valency 1 . We first associate a weight $\ell_{v w} \in \mathbb{N}$ to a pair consisting of a node $v$ and end $w$. The weight $\ell_{v w}$ is the product of the edge weights in $\Delta$ that are adjacent to, but not on, the path from $v$ to $w$ in $\Delta$. Let $\ell_{v w}^{\prime}$ be the same product but excluding weights adjacent to $v$ itself. Thus,

$$
\ell_{v w} d_{v e}=\ell_{v w}^{\prime} d_{v}
$$

where $d_{v}$ is the product of edge weights adjacent to $v$ and $e$ is the first edge of the path from $v$ to $w$.

Example 1. For example, in the above example with vertices labelled as follows

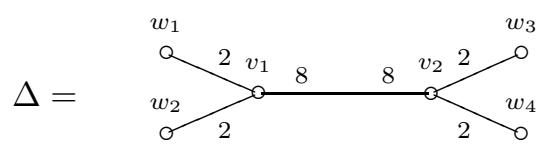

we have $\ell_{v_{1} w_{1}}=16, \ell_{v_{1} w_{1}}^{\prime}=1, \ell_{v_{1} w_{3}}=8, \ell_{v_{1} w_{3}}^{\prime}=2$, etc.

We are interested in a numerical condition on the splice diagram. 
Conjecture 2 (Semigroup condition). Let $\Delta$ be the splice diagram associated to a $\mathbb{Q}$-Gorenstein singularity with $\mathbb{Q}$-homology sphere link. Then for each node $v$ and adjacent edge e the edge-weight $d_{v e}$ is in the semigroup

$$
\mathbb{N}\left\langle\ell_{v w}^{\prime}: w \text { an end of } \Delta \text { in } \Delta_{v e}\right\rangle,
$$

where $\Delta_{v e}$ is the subtree of $\Delta$ cut off by $e$.

Equivalently, if $d_{v}$ is the product of the edge weights $d_{v e}$ adjacent to $v$, then

$$
d_{v} \in \mathbb{N}\left\langle\ell_{v w}: w \text { an end of } \Delta \text { in } \Delta_{v e}\right\rangle
$$

The condition of this conjecture is not true for every resolution tree. In particular, this Conjecture would imply a highly non-trivial (and unexpected) topological condition for a singularity to be $\mathbb{Q}$-Gorenstein. It is shown in [11] that this condition is implied if certain naturally defined knots in the link $\vec{M}$ are cut out by hyperplane sections of $(X, o)$. The content of Conjecture 2 is that this condition is necessary for the tree to be realizable by a $\mathbb{Q}$-Gorenstein singularity.

So, let us now consider a splice diagram $\Delta$ satisfying the semigroup condition (such as our example above). The associated complete intersection singularity will have embedding dimension less than or equal to the number $n$ of ends of $\Delta$. Thus, to each end $w$ we associate a variable $z_{w}$.

To each node $v$ of $\Delta$ of valency $\delta_{v}$ we will associate $\delta_{v}-2$ equations. It is easy to check that $n-2=\sum\left(\delta_{v}-2\right)$ (summed over the nodes of $\Delta$ ), so this will give the right number of equations.

We describe the $\delta_{v}-2$ equations associated to a node $v$. For each end $w$ we give the variable $z_{w}$ weight $\ell_{v w}$ (we call this the $v$-weight of $z_{w}$ ). To each edge $e$ at $v$ we can now associate one or more admissible monomials $\prod_{w} z_{w}^{\alpha_{v w}}$, product over ends $w$ in $\Delta_{v e}$, with the exponents $\alpha_{v w} \in \mathbb{N}$ satisfying

$$
d_{v}=\sum_{w} \alpha_{v w} \ell_{v w}, \quad \text { sum over } w \text { an end in } \Delta_{v e} .
$$

Thus each admissible monomial has total $v$-weight $d_{v}$. Each of the $\delta_{v}-2$ equations associated to $v$ is obtained by equating to zero some $\mathbb{C}$-linear combination of the admissible monomials associated to $v$. If $\Delta$ has just one node this gives equations of Brieskorn-Pham type.

We choose $\delta_{v}-2$ equations in this way for each node $v$, giving a total of $n-2$ equations in the $n$ variables $z_{w}$. We must also require that the coefficients of the equations are "sufficiently general" in an appropriate sense.

Definition 2.2. We say such a system of equations is in splice diagram form.

We will not specify in detail what "sufficiently general" means here. It is a generic condition which usually depends on all the coefficients at once. But if, for some node $v$, we have chosen just one admissible monomial for each edge at $v$, and the $\delta_{v}-2$ equations associated to $v$ involve only these $\delta_{v}$ monomials, then "sufficiently general" for these equations means that all maximal minors of the $\left(\delta_{v}-2\right) \times \delta_{v}$ matrix of coefficients are non-singular. If $\Delta$ has one node (Brieskorn-Pham complete intersection) this is the well-known condition for an isolated singularity due to $\mathrm{H}$. Hamm.

Thus, for splice diagrams with one node, "splice diagram form" gives exactly the isolated Brieskorn-Pham complete intersection singularities. 
Example 1 (ctd.). For the $\Delta$ of Example1 we abbreviate $z_{w_{i}}=z_{i}$. The admissible monomials for vertex $v_{1}$ are $z_{1}^{2}, z_{2}^{2}$, and any $z_{3}^{\alpha} z_{4}^{\alpha^{\prime}}$ with $2 \alpha+2 \alpha^{\prime}=8$. Thus the equation associated to the node $v_{1}$ might be $z_{1}^{2}+z_{2}^{2}+z_{3}^{\alpha} z_{4}^{4-\alpha}=0$, for some $0 \leq \alpha \leq 4$. Similarly for the second node, so our system of equations might be

$$
\begin{aligned}
& z_{1}^{2}+z_{2}^{2}+z_{3}^{\alpha} z_{4}^{4-\alpha}=0, \\
& z_{3}^{2}+z_{4}^{2}+z_{1}^{\beta} z_{2}^{4-\beta}=0 .
\end{aligned}
$$

This system is always in "splice diagram form" by our comments above. However, if we replace the equations, for example, by $a z_{1}^{2}+b z_{2}^{2}+z_{3}^{\alpha} z_{4}^{4-\alpha}=0$ and $z_{3}^{2}+z_{4}^{2}+$ $c z_{1} z_{2}^{3}+d z_{1}^{3} z_{2}=0$, then the condition that the coefficients are sufficiently general is: $a b \neq 0$ and $a d-b c \neq 0$.

Theorem 2.3. A system of equations in splice diagram form defines an isolated complete intersection surface singularity.

We would like a complete intersection of this form to give the universal abelian cover of our original singularity (up to equisingularity, actually). Since the covering transformation group will be the discriminant group of $A(\Gamma)$, this group should act in a natural way on the n-dimensional space of the variables $z_{w}$. This is indeed the case:

Proposition 2.4. The discriminant group of $A(\Gamma)$ acts faithfully and diagonally on the $n$-dimensional space of the variables $z_{w}$, and acts freely in codimension 1 (that is, no coordinate hyperplane is fixed by a non-trivial group element).

The action is generated by the diagonal matrices

$$
\left[\exp \left(2 \pi i \bar{a}_{w_{j} w_{1}}\right), \ldots, \exp \left(2 \pi i \bar{a}_{w_{j} w_{n}}\right)\right]
$$

for $j=1, \ldots, n$, where $\bar{a}_{w w^{\prime}}$ is the $\left(w, w^{\prime}\right)$-entry of the inverse matrix $A(\Gamma)^{-1}$.

In order for this group to act on the complete intersection singularity, one needs to be able to choose the equations in an appropriate way. It turns out that this is sometimes not possible, even if $\Delta$ satisfies the semigroup condition. One obtains a new set of numerical conditions, which we call the congruence conditions; these will guarantee that the group acts freely (off the origin) on the complete intersection singularity. Since the action of the discriminant group depends not only on the splice diagram, but on the resolution graph, the congruence conditions depend on the resolution graph.

Conjecture 3. The resolution graph of a $\mathbb{Q}$-Gorenstein singularity with $\mathbb{Q}$-homology sphere link satisfies the congruence conditions.

Example 1 (ctd.). Returning to our example above, the discriminant group is a group of order 16 whose action on $\mathbb{C}^{4}$ is generated by the four diagonal matrices

$$
[1,-1, i, i],[-1,1, i, i],[i, i, 1,-1],[i, i,-1,1] \text {. }
$$

If this action is to preserve the variety given by equations (2), the exponents $\alpha$ and $\beta$ must be odd. This is the congruence condition in this case. This example is one of a class of examples (quotient-cusps) for which we have verified our conjectures. 
Examples 2. For the following example the splice diagram does not satisfy the semigroup condition:

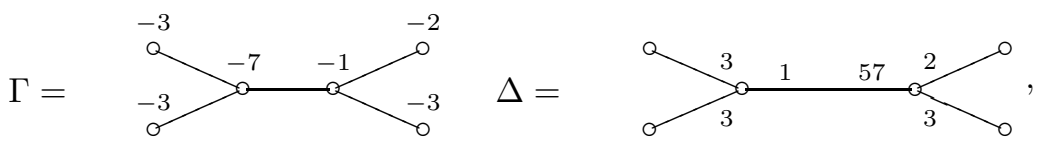

since 1 is not in the semigroup generated by 2 and 3 . For the following example the splice diagram satisfies the semigroup condition, but the resolution graph does not satisfy the congruence condition:

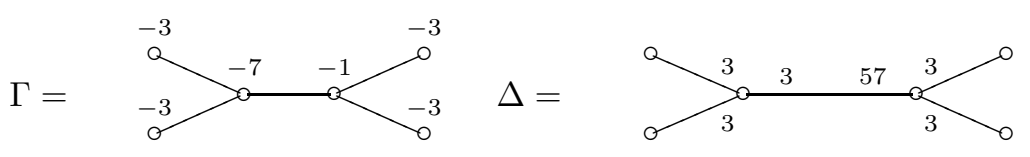

There are two important classes of singularities that are topologically determined to be $\mathbb{Q}$-Gorenstein singularities with $\mathbb{Q}$-homology sphere links: the rational singularities and (with two classes of exceptions) the minimally elliptic singularities of Laufer [3]. In the first non-trivial case we have verified that the above purportedly necessary numerical conditions on the graph are indeed satisfied.

Theorem 2.5. Let $(X, o)$ be a rational or minimally elliptic singularity (not simple elliptic or a cusp), whose resolution graph has two nodes (vertices of valency at least 3). Then the splice diagram of the resolution graph satisfies the semigroup condition, and the congruence conditions are satisfied as well.

When the semigroup and congruence conditions are satisfied, one should show that the quotient of the splice diagram singularity by the group action has the same topological type as $(X, o)$, but we have not yet proved this.

We have a natural notion of equisingular deformation of the above complete intersections. We may add to each equation a linear combination of monomials which for each node $v$ have $v$-weight greater than or equal to $d_{v}$, and so that the discriminant group continues to transform each equation by a character. These ought to give equisingular deformations of the original quotient. In the case of one node, when dealing with Brieskorn-Pham complete intersections, these are the deformations of weight $\geq 0$. Our final Conjecture will say that all $\mathbb{Q}$-Gorenstein singularities with $\mathbb{Q}$-homology sphere link arise in this way.

Conjecture 4. Let $(X, o)$ be a $\mathbb{Q}$-Gorenstein singularity with $\mathbb{Q}$-homology sphere link. Let $\Delta$ be the splice diagram associated to the resolution graph. Then $\Delta$ satisfies the semigroup condition, the resolution graph satisfies the congruence condition, and the universal abelian cover of $(X, o)$ is an equisingular deformation of the complete intersection of splice diagram form defined above. The geometric genus of the singularity is determined by the topology.

For the last assertion concerning the geometric genus, there are some deep partial results due to A. Némethi in [5].

\section{Results And EVIDEnCE}

We have verified the conjectures for several examples. The evidence for our conjectures also includes phenomena that seem to hold in more general situations than those described by the conjectures. We describe now some of this evidence. 
3.1. The topology of the universal abelian cover is determined by the splice diagram. Although a resolution tree determines a splice diagram, the resolution tree cannot be recovered from the splice diagram. If the splice diagram has just one node (this is the situation of Theorem 1.1) then there are infinitely many resolution trees sharing the same splice diagram; otherwise just finitely many. If our conjectures are correct then the homeomorphism type of the universal abelian cover of the link of a $\mathbb{Q}$-Gorenstein singularity with $\mathbb{Q}$-homology sphere link should only depend on the splice diagram of the resolution tree. This is indeed true, even without the $\mathbb{Q}$-Gorenstein assumption:

Theorem 3.1. If two singularities whose links are $\mathbb{Q}$-homology spheres have the same splice diagram then the universal abelian covers of their links are diffeomorphic.

3.2. Quotient-cusps. We have succeeded in proving our conjectures in a number of cases. The quasihomogeneous case was already known by [8]. A new example is treated in 10]. Among the log-canonical surface singularities, the only non-weighted homogeneous $\mathbb{Q}$-homology sphere link singularities are the quotient-cusps. These are $\mathbb{Z} / 2$ quotients of certain cusp singularities; so it is obvious that the universal abelian cover is also a cusp. Given a resolution graph of a cusp, it is easy to tell if it is a complete intersection, by an old result of Karras. But it requires some extensive argument to show that in our situation this always occurs. To actually write down the equations of the universal abelian cover, and explicit action of the discriminant group, was originally an extremely difficult task. But the discovery of a simpler way to do it led to the description of the generalized Brieskorn-Pham equations plus diagonal group action considered above.

3.3. Integral homology sphere links. In the special case that the link $M$ of our singularity is an integral homology sphere, that is $H_{1}(M ; \mathbb{Z})=\{0\}$, our conjectures become:

Conjecture 5. Let $(X, o)$ be a Gorenstein normal surface singularity whose link is an integral homology sphere. Then $X$ is a complete intersection singularity, its splice diagram $\Delta$ satisfies the semigroup condition, and its equations are equisingular deformations of those in Section 2. The Casson invariant of the link is one-eighth the signature of the Milnor fibre of X (cf. [9]).

For a splice diagram with two nodes that represents an integral homology sphere, as well as some other cases, we can show that our equations define a singularity whose link has the correct topological type, and for which the Casson invariant is as conjectured [1].

\section{REFERENCES}

[1] D. Eisenbud and W. D. Neumann, Three-dimensional link theory and invariants of plane curve singularities. Ann. Math. Stud. 110, Princeton. Princeton Univ. Press (1985).

[2] F. Klein, Lectures on the Icosahedron and the Solution of Equations of the Fifth Degree, Translated by George Gavin Morrice. Second andrevised ed. (Dover Publications, Inc., New York, NY, 1956).

[3] H. B. Laufer, On minimally elliptic singularities. Amer. J. Math. 99 (1977), 1257-1295.

[4] J. Milnor, On the 3-dimensional Brieskorn manifold $M(p, q, r)$, Papers dedicated to the memory of R.H. Fox, Ann. Math. Studies 84, 175-225 (Princeton U. Press, 1975)

[5] A. Némethi, Weakly elliptic Gorenstein singularities of surfaces, Invent. Math. 137 (1999), 145-167. 
[6] W.D. Neumann, Brieskorn complete intersections and automorphic forms, Invent. Math. 42 (1977) 285-293.

[7] _ A calculus for plumbing applied to the topology of complex surface singularities and degenerating complex curves, Trans. Amer. Math. Soc. 268 (1981), 299-343.

[8] Abelian covers of quasihomogeneous surface singularities, Singularities, Arcata 1981, Proc. Symp. Pure Math. 40 (Amer. Math. Soc. 1983), 233-243.

[9] W.D. Neumann, J. Wahl, Casson invariant of links of singularities, Comment. Math. Helv. 65 (1990), 58-78.

[10] - Universal abelian covers of quotient-cusps. (preprint).

[11] Complete intersection singularities with integral homology sphere links (in preparation).

Department of Mathematics, Barnard College, Columbia University, New York, Ny 10027

E-mail address: neumann@math.columbia.edu

Department of Mathematics, The University of North Carolina, Chapel Hill, NC 27599-3250

E-mail address: jw@math.unc.edu 